\title{
The GRB 060218/SN 2006aj event in the context of other gamma-ray burst supernovae ${ }^{\star, \star \star}$
}

\author{
P. Ferrero ${ }^{1}$, D. A. Kann ${ }^{1}$, A. Zeh², S. Klose ${ }^{1}$, E. Pian ${ }^{2}$, E. Palazzi ${ }^{3}$, N. Masetti ${ }^{3}$, D. H. Hartmann ${ }^{4}$, \\ J. Sollerman ${ }^{5}$, J. Deng ${ }^{6}$, A. V. Filippenko ${ }^{7}$, J. Greiner ${ }^{8}$, M. A. Hughes ${ }^{9}$, P. Mazzali ${ }^{2}, 10$, \\ W. Li ${ }^{7}$, E. Rol ${ }^{11}$, R. J. Smith ${ }^{12}$, and N. R. Tanvir ${ }^{9,11}$ \\ 1 Thüringer Landessternwarte Tautenburg, 07778 Tautenburg, Germany \\ e-mail: ferrero@tls-tautenburg.de \\ 2 Istituto Nazionale di Astrofisica-OATs, 34131 Trieste, Italy \\ 3 INAF, Istituto di Astrofisica Spaziale e Fisica Cosmica, Sez. di Bologna, 40129 Bologna, Italy \\ 4 Clemson University, Department of Physics and Astronomy, Clemson, SC 29634-0978, USA \\ 5 Dark Cosmology Center, Niels Bohr Institute, Copenhagen University, 2100 Copenhagen, Denmark \\ ${ }^{6}$ National Astronomical Observatories, CAS, Chaoyang District, Beijing 100012, PR China \\ 7 Department of Astronomy, University of California, Berkeley, CA 94720-3411, USA \\ 8 Max-Planck-Institut für extraterrestische Physik, 85741 Garching, Germany \\ 9 Centre for Astrophysics Research, University of Hertfordshire, College Lane, Hatfield, AL10 9AB, UK \\ ${ }_{10}$ Max-Planck Institut für Astrophysik, 85748 Garching, Germany \\ 11 Department of Physics and Astronomy, University of Leicester, Leicester, LE1 7RH, UK \\ 12 Astrophysics Research Institute, Liverpool John Moores University, Twelve Quays House, Birkenhead, CH41 1LD, UK \\ Received 2 May 2006 / Accepted 13 July 2006
}

\section{ABSTRACT}

\begin{abstract}
The supernova SN 2006aj associated with GRB 060218 is the second-closest GRB-SN observed to date $(z=0.033)$. We present Very Large Telescope, Liverpool Telescope, and Katzman Automatic Imaging Telescope multi-color photometry of SN 2006aj. This supernova is found to be subluminous and rapidly evolving. Its early light curve includes an additional wavelength-dependent component, which can be interpreted as shock break-out. We compare the photometric evolution of multi-band light curves with the corresponding properties of the present sample of more than $10 \mathrm{GRB}-\mathrm{SNe}$ with precisely known redshifts. Using host-galaxy extinction measurements, we derive extinction-corrected GRB-SN luminosities and place SN 2006aj in the context of this GRB-selected supernova sample as well as in the context of local stripped-envelope supernovae.
\end{abstract}

Key words. gamma rays: bursts: X-rays: individuals: GRB 060218 - supernovae: individual: SN 2006aj

\section{Introduction}

There is now substantial evidence for the association of longduration GRBs with core-collapse supernovae (SNe of type Ic to be more specific; see Filippenko 1997 for a review of supernova spectral classification). The first hint for such a relation came with the contemporaneous discovery of GRB 980425 and of a local SN (1998bw) in its error circle (Galama et al. 1998). Subsequently, Bloom et al. (1999) reported a possible underlying SN component in the afterglow of GRB 980326. The first spectroscopic identification of a SN Ic superposed on a GRB afterglow (GRB 030329/SN 2003dh; Hjorth et al. 2003; Matheson et al. 2003; Stanek et al. 2003) solidified this association considerably.

The photometric database on GRB-SNe was summarized by Zeh et al. (2004, 2005, henceforth Z04, Z05) who, by studying a sample of 21 (Z04) and 29 (Z05) bursts with well established redshifts in a systematic way, found or confirmed a weak SN component for 9 (Z04) and 13 (Z05) sources in

* Based on observations collected at the European Southern Observatory, Paranal, Chile (ESO Programme 076.D-0015).

$\star \star$ Table 1 is only available in electronic form at http://www . edpsciences. org. those sets, respectively. In particular, all long bursts with redshifts $z \lesssim 0.7$ were found to have a late-time bump in their optical afterglows, suggesting that in fact all long bursts are physically associated with SN explosions. Additional photometric and partly spectroscopic results on recent GRB-SNe with well-known redshift were presented by Malesani et al. (2004; GRB 031203/SN 20031w), Soderberg et al. (2006) and Stanek et al. (2005) on SNe associated with GRB 040924 and GRB 041006, as well as Della Valle et al. (2006; GRB 050525A/SN 2005nc). A recent review of the status of the supernova - GRB connection is presented by Woosley \& Bloom (2006).

GRB 980425/SN 1998bw is still the closest GRB-SN to date, and poses the question whether it is a typical representative of this class, a "standard source" in some sense. Its comparatively low isotropic energy release in the gamma-ray band (less than $10^{48} \mathrm{erg}$, Galama et al. 1998) makes this burst stand out from the cosmological GRB ensemble with known redshifts (cf. Ghirlanda et al. 2004). It is therefore of great interest to find additional events in the nearby universe. Consequently, the discovery of GRB 060218 (Cusumano et al. 2006) (more precisely an X-Ray Flash, XRF) and its identification with an energetic SN by Masetti et al. (2006), now designated SN 2006aj 
(Soderberg et al. 2006), attracted much attention (Modjaz et al. 2006a; Sollerman et al. 2006; Mirabal et al. 2006; Cobb et al. 2006). Its small distance $(z=0.033$, Mirabal \& Halpern 2006) results in an isotropic equivalent energy of the burst of $5 \times 10^{49}$ erg in the $15.5-154.8 \mathrm{keV}$ band, which again is underenergetic in comparison to the cosmological GRB sample (Modjaz et al. 2006a), whose mean redshift is larger than $z=1$ (Jakobsson et al. 2006; Bagoly et al. 2006).

Here, we report results from a photometric ESO Very Large Telescope (VLT) campaign on GRB 060218/SN 2006aj covering a time span of nearly 4 weeks, with additional data gathered with the robotic Liverpool Telescope (LT) at La Palma and the robotic Katzman Automatic Imaging Telescope (KAIT; Filippenko et al. 2001; Li et al. 2003a) at Lick Observatory. Results from the spectroscopic VLT campaign are presented in Pian et al. (2006) and modeled in Mazzali et al. (2006b). While previous publications on SN 2006aj discussed the X-ray and optical light curves in detail, we place the photometric evolution of SN 2006aj in the context of all presently known GRB-SNe with precise redshifts. In particular, we update the fits reported in Z04 and Z05, add several more recent GRB-SNe, and correct the photometry for host-galaxy extinction as derived in Kann et al. (2006, henceforth K06).

\section{Observations and data reduction}

\subsection{VLT data}

We observed SN 2006aj both spectroscopically and photometrically with the ESO Very Large Telescope FORS1 and FORS2 instruments. Observations were performed until 26 days after the burst (Table 1). After this point in time, the SN location was no longer observable due to high airmass. For the photometry the exposure time was between 30 and 60 seconds. The images, in the Bessell $B, V, R$, and $I$ filters, were bias-subtracted and flat-fielded with standard reduction procedures using IRAF ${ }^{1}$ and final photometry was performed with standard Point Spread Function (PSF) fitting using the DAOPHOT II image data analysis package (Stetson 1987) within MIDAS ${ }^{2}$. Photometric calibration of the images was performed using the standard star 685 of the field SA98 (Landolt 1992), imaged during the same night of our first observational run, which was used to create 16 secondary standards in the field around the SN that were subsequently used for all individual images to draw a linear regression among instrumental and reference magnitudes and calculate the value of the optical transient. The accuracy of the photometry was confirmed by considering the zero point values of several stars in the calibration field in all bands for different nights. In addition, we investigated a possible influence of color terms. We found that they could affect the photometry with an additional error of up to $0.02 \mathrm{mag}$, which was then added in quadrature to the individual photometric measurement errors.

\subsection{Liverpool Telescope data and KAIT data}

Further photometric data were obtained with the $2 \mathrm{~m}$ robotic Liverpool Telescope on La Palma on several occasions over a period of about 2.5 weeks post-burst. Observations were made in Bessell $B, V$ and SDSS $r^{\prime}$ filters and zero-point reduced by comparison to two field stars (star S1 and star 4 of Modjaz et al. 2006a). The magnitudes were transformed into Johnson $B, V$ and

\footnotetext{
1 http://iraf.noao.edu

2 http://www . eso.org/projects/esomidas
}

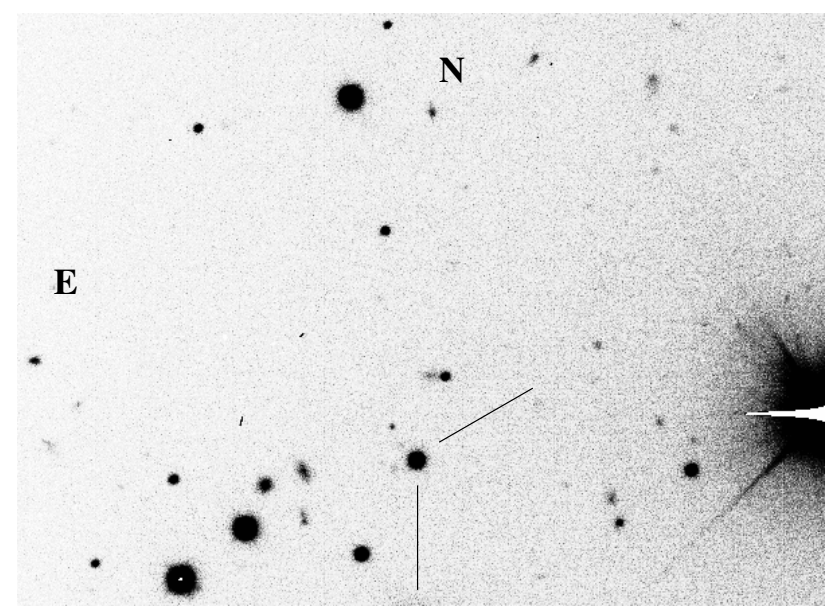

Fig. 1. The location of SN 2006aj is shown here in a VLT/FORS2 $R$ band image taken on 2006 March 1, at 00:52 UT (30 s exposure time). The field of view is approximately $2 \times 3 \mathrm{arcmin}$.

Cousins $R$-band via equations previously derived for the camera (Steele 2004). The quoted errors reflect the scatter in individual measurements for subexposures at each epoch, and include an estimate of the calibration uncertainty.

Additional data were also obtained with the $0.8 \mathrm{~m}$ robotic KAIT at Lick Observatory on four consecutive nights between 3 to 6 days post-burst. Unfiltered observations were obtained in all four nights, and Bessell $B, V, R$, and $I$ filtered observations were made in the last three nights. The images were bias-subtracted and flat-fielded with standard reduction procedures using IRAF, and the final photometry was performed with PSF-fitting technique in IRAF/DAOPHOT. The secondary standards calibrated with VLT data were used for the final photometric calibration. The unfiltered magnitudes were converted to the $R$ band following the method described in Li et al. (2003b).

\subsection{The magnitudes of the host galaxy}

The host galaxy of GRB 060218/SN 2006aj was imaged preburst by the Sloan Digital Sky Survey (Cool et al. 2006; Adelman-McCarthy et al. 2006). It was pointed out (Modjaz et al. 2006a; Hicken et al. 2006) that there are offsets between the SDSS calibration and later efforts, rendering the transformed host-galaxy magnitudes of Cool et al. (2006) too bright. The values used for the host galaxy in this paper are $B=20.57 \pm 0.07$, $V=20.18 \pm 0.04, R=20.03 \pm 0.03$ and $I=19.58 \pm 0.06$, not corrected for Galactic extinction. These magnitudes have been evaluated from those reported by Cool et al. (2006), by applying the corrections recommended by Modjaz et al. (2006a) and Hicken et al. (2006). Our BVRI magnitudes have been obtained from Cool et al.'s $u^{*} b^{*} v^{*} r^{*} i^{*}$ magnitudes by adopting the conversion of Lupton $(2005)^{3}$ and taking into account that the offsets determined by Modjaz et al. (2006) in the $B V R$ bands are 0.40 , $0.27,0.20 \mathrm{mag}$, respectively ${ }^{4}$. In order to determine the offset in the $I$ band, we have considered that the offset determined by Hicken et al. (2006) and Modjaz et al. (2006a) in the $i^{*}$-filter is $0.15 \mathrm{mag}$, and we have used the SDSS transformations ${ }^{5}$ to

\footnotetext{
3 See also http://www. sdss.org/dr4/algorithms/sdssUBVRI Transform.html\#Rodgers2005

4 See http://www.cfa.harvard.edu/oir/Research/ supernova/sn2006aj/compstars.html

5 See http://www.sdss.org/dr4/algorithms/ jeg_photometric_eq_dr1.html\#usno2SDSS
} 
derive from this the offset in the $i$-band. We note that our hostgalaxy magnitudes are consistent to those of Sollerman et al. (2006) within the errors. As the host galaxy and SN 2006aj were not separated at the resolution of the images, the host magnitudes have to be subtracted.

\subsection{The extinction correction}

For the extinction correction, we used the values derived by Guenther et al. (2006) from an analysis of the Na I D2 absorption lines in our Galaxy $\left(A_{\mathrm{V}}=0.39 \mathrm{mag}\right)$ and the host galaxy $\left(A_{\mathrm{V}}=0.13 \mathrm{mag}\right)$ in a VLT UVES spectrum, obtained during this campaign close to SN maximum. We note that this host-galaxy extinction is typical of the lines of sight toward GRBs (K06). Using these extinction and host magnitude values, we derive the magnitudes of the pure SN light component.

\section{Results}

We followed previous works (Z04, Z05) and modeled the light curve of SN2006aj using SN 1998bw as a template (Galama et al. 1998) that was shifted ${ }^{6}$ to the corresponding redshift and scaled in luminosity (in the SN rest frame) by a factor $k$ and in time evolution by a factor $s$, while zero host extinction was assumed for SN 1998bw (Patat et al. 2001). In doing so, we find an additional component visible in the early data that makes the light curve systematically brighter than that of SN 1998bw. This component has also been noted by Mirabal et al. (2006). Furthermore, strong spectral evolution is noticeable, with the flux excess being stronger toward shorter wavelengths. The most reasonable explanation for this additional blue component is the light due to shock break-out through a dense progenitor wind (Campana et al. 2006). However, we caution that at least part of this additional component may be the result of an intrinsically different (as compared to SN 1998bw) early SN light curve. SN 2002ap (cf. Appendix) is also overluminous at early times in comparison to SN 1998bw, and has been compared, both photometrically and spectroscopically, with SN 2006aj (e.g. Pian et al. 2006). But the early multi-color evolution of SN 2002ap is achromatic, showing no evidence for strong spectral evolution as seen in SN 2006aj.

We thus used two different methods to fit the SN light curve. In the first fit, we excluded all data earlier than 8.8 days, but fit only the SN 1998bw model light curve to the data, without any additional contributions. The result is shown in Fig. 2. The early excess blue flux is clearly visible in the residuals. The data after 8.8 days are matched very well by the SN 1998 bw model. Note that for typical GRB-SNe we also fit only the peak and decaying part of the $\mathrm{SN}$, as the early flux is typically dominated by the GRB afterglow.

In the second fit, we assumed that an additional component decays according to a power-law, with the decay index $\alpha$ $\left(F_{v} \propto t^{-\alpha}\right)$ being a free parameter of the fit, and independent for each band (Fig. 3). The derived values of the luminosity ratio $k$ and the stretch factor $s$ as well as the decay index $\alpha$ are given in Table 2. It is apparent that while the results derived from the two methods are similar, they do in fact differ by as much as $10 \%$ (in $k$ ). The additional component improves the quality of the fit in all bands. The steeper decay at shorter wavelengths is also in agreement with the Swift UVOT photometry of the blue shock

${ }^{6}$ Throughout the paper we adopt $H_{0}=71 \mathrm{~km} \mathrm{~s}^{-1} \mathrm{Mpc}^{-1}, \Omega_{\mathrm{M}}=0.27$, $\Omega_{\Lambda}=0.73$ (Spergel et al. 2003), which for $z=0.033$ yields a distance modulus of $35.78 \mathrm{mag}$.

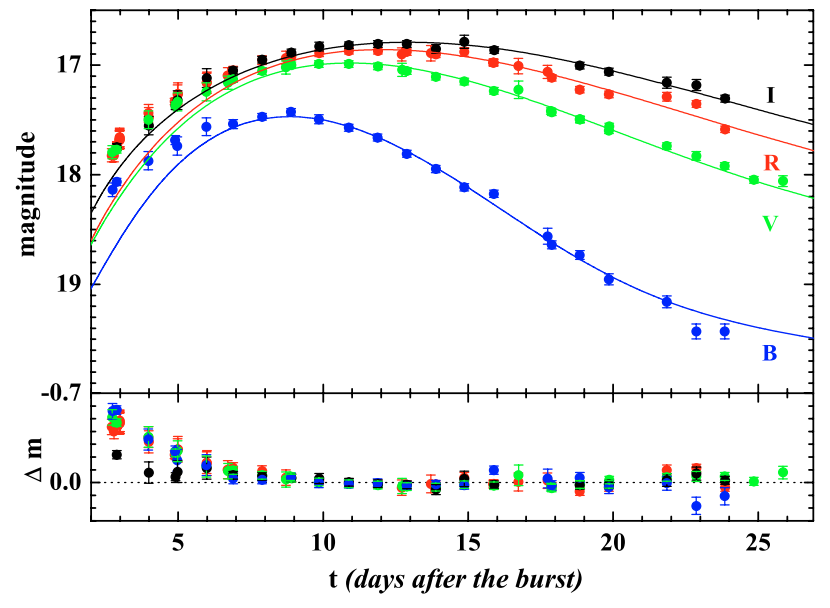

Fig. 2. The light curves of SN 2006aj based on our BVRI data after correcting for extinction and host flux contribution. The data were fitted using the light curves of SN 1998bw as a template, not considering data from SN 2006aj that was taken before 8.8 days post burst. The residuals $\Delta m$ represent observed values minus the fit.

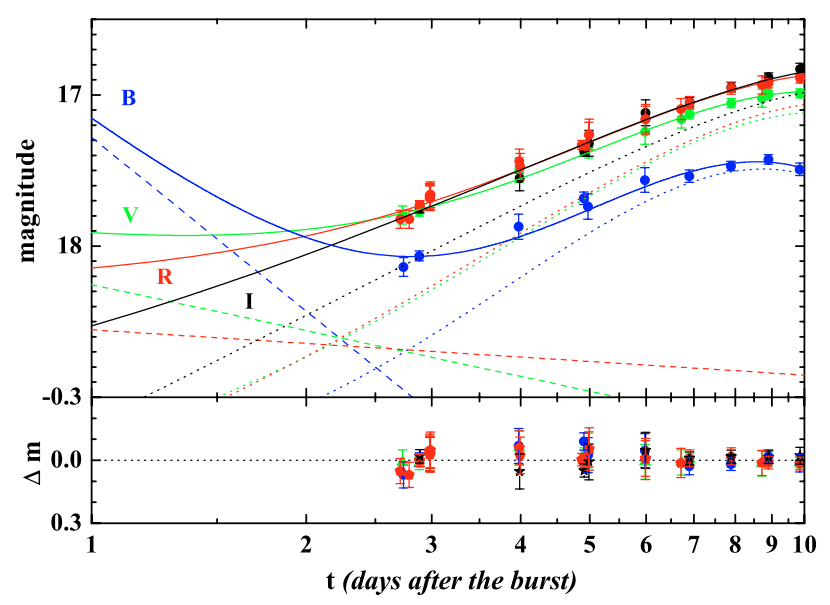

Fig. 3. The light curves of SN 2006aj fitted by including an additional early component which decays according to a power-law. Solid lines mark the fit, the dashed lines are the power-law component and the dotted lines are the SN component. The time scale is logarithmic to show in detail the earlier data.

break-out (Campana et al. 2006). The strong wavelength dependence of the decay (Table 2) argues against this component being the optical afterglow of the GRB. On the other hand, the very shallow decay in $V R I$ indicates that the time evolution of the SED of this additional component is probably more complex than a simple decay according to a pure power-law, as assumed in our analysis.

The small value of the stretch factor $s$ means that SN 2006aj evolves much faster than SN 1998bw (see also Pian et al. 2006; Modjaz et al. 2006a; Sollerman et al. 2006; Mirabal et al. 2006; Cobb et al. 2006; Soderberg et al. 2006). Furthermore, SN 2006aj has a slightly different color than SN 1998bw, being brighter in the $B$ and $I$ band than in the $V$ and $R$ band. The $k$ values we obtain are also in accordance with the values found by the authors cited above. In all bands, SN 2006aj is about 30\% less luminous than SN 1998bw. From the light curve data, independent of any fit, we find the following peak absolute magnitudes of the $\mathrm{SN}: M_{B}=-18.29 \pm 0.05, M_{V}=-18.76 \pm 0.05$, $M_{R}=-18.89 \pm 0.05, M_{I}=-18.95 \pm 0.10$. 
Table 2. Fitting results for the light curves of SN 2006aj attained by either excluding early data (top) or including an additional power-law component decaying with index $\alpha$ (see text for details).

\begin{tabular}{ccccr}
\hline \hline & $\chi_{v}^{2}$ & $k$ & $s$ & $\alpha$ \\
\hline$B$ & 1.83 & $0.734 \pm 0.014$ & $0.620 \pm 0.006$ & $\cdots$ \\
$V$ & 1.02 & $0.724 \pm 0.007$ & $0.682 \pm 0.005$ & $\cdots$ \\
$R$ & 1.93 & $0.735 \pm 0.007$ & $0.689 \pm 0.006$ & $\cdots$ \\
$I$ & 0.71 & $0.761 \pm 0.008$ & $0.686 \pm 0.008$ & $\cdots$ \\
\hline$B$ & 1.79 & $0.721 \pm 0.022$ & $0.613 \pm 0.005$ & $1.53 \pm 0.43$ \\
$V$ & 0.35 & $0.640 \pm 0.014$ & $0.659 \pm 0.008$ & $0.40 \pm 0.09$ \\
$R$ & 0.94 & $0.621 \pm 0.022$ & $0.656 \pm 0.012$ & $0.12 \pm 0.10$ \\
$I$ & 0.48 & $0.667 \pm 0.039$ & $0.663 \pm 0.022$ & $-0.10 \pm 0.34$ \\
\hline
\end{tabular}

Table 3. Luminosity ratio $k$ and stretch factor $s$ for GRB-SNe with known redshift normalized to SN 1998bw before and after correcting for host extinction. In cases where no extinction could be derived, only the non-corrected values are given. In all cases the SN fits were derived in the observer $R$-band frame. This list is complete up to the end of 2005, with the exception of GRB 040924 (Soderberg et al. 2006). These results supersede those presented in Z05. Differences compared to Z05 are basically due to the inclusion of new observational data or a revision of the used value for the Galactic extinction along the line of sight. Furthermore, note that we use different cosmological parameters than those in Z04 and Z05.

\begin{tabular}{lccc}
\hline \hline GRB/XRF/SN & $k$ & $s$ & $k$ (corrected) \\
\hline 970228 & $0.40 \pm 0.29$ & $1.45 \pm 0.95$ & $\ldots$ \\
990712 & $0.35 \pm 0.09$ & $0.83 \pm 0.13$ & $\ldots$ \\
991208 & $0.93 \pm 0.25$ & $1.12 \pm 0.20$ & $2.62_{-0.65}^{+1.10}$ \\
000911 & $0.63 \pm 0.29$ & $1.40 \pm 0.32$ & $0.85_{-0.26}^{+0.26}$ \\
010921 & $0.68 \pm 0.43$ & $0.69 \pm 0.25$ & $1.85_{-0.79}^{+2.82}$ \\
$011121 / 2001 \mathrm{ke}$ & $0.59 \pm 0.02$ & $0.80 \pm 0.02$ & $0.88_{-0.07}^{+0.08}$ \\
020405 & $0.74 \pm 0.05$ & $0.97 \pm 0.07$ & $0.90_{-0.11}^{+0.15}$ \\
020903 & $0.62 \pm 0.09$ & $0.92 \pm 0.08$ & $\ldots$ \\
$021211 / 2002 \mathrm{lt}$ & $0.40 \pm 0.19$ & $0.98 \pm 0.26$ & $\ldots$ \\
$030329 / 2003 \mathrm{dh}$ & $1.06 \pm 0.11$ & $0.85 \pm 0.10$ & $1.50_{-0.16}^{+0.19}$ \\
$031203 / 20031 \mathrm{w}$ & $0.67 \pm 0.05$ & $1.09 \pm 0.07$ & $1.28_{-0.16}^{+0.16}$ \\
041006 & $0.90 \pm 0.05$ & $1.38 \pm 0.06$ & $1.03_{-0.09}^{+0.22}$ \\
$050525 \mathrm{~A} / 2005 \mathrm{nc}$ & $0.49 \pm 0.04$ & $0.77 \pm 0.04$ & $0.66_{-0.08}^{+0.10}$ \\
\hline
\end{tabular}

In order to place SN 2006aj in the context of the presently known GRB-SNe, we started with the results presented in Z04 and $\mathrm{Z05}$, updated them in cases where new data were available, and corrected the $k$ value using host-galaxy extinctions derived in K06, including uncertainties. We added two further GRB-SNe to the sample. The result for XRF 020903 is based on data from Bersier et al. (2006). We found that the SN of this event is also superimposed on an underlying power-law afterglow component. Unfortunately, the afterglow data are too sparse to derive any conclusions on the extinction in the host galaxy. For GRB 031203, we used the extinction derived by Mazzali et al. (2006a). In the case of GRB 050525A, we employed the data presented by Della Valle et al. (2006) and applied the extinction found by Blustin et al. (2006). In those cases where no extinction could be found in K06, we only present the observed values and note that these are lower limits on the luminosity of the SNe (Table 3 ). On the other hand, the line of sight extinction is often low and the true values are thus not expected to be much higher.

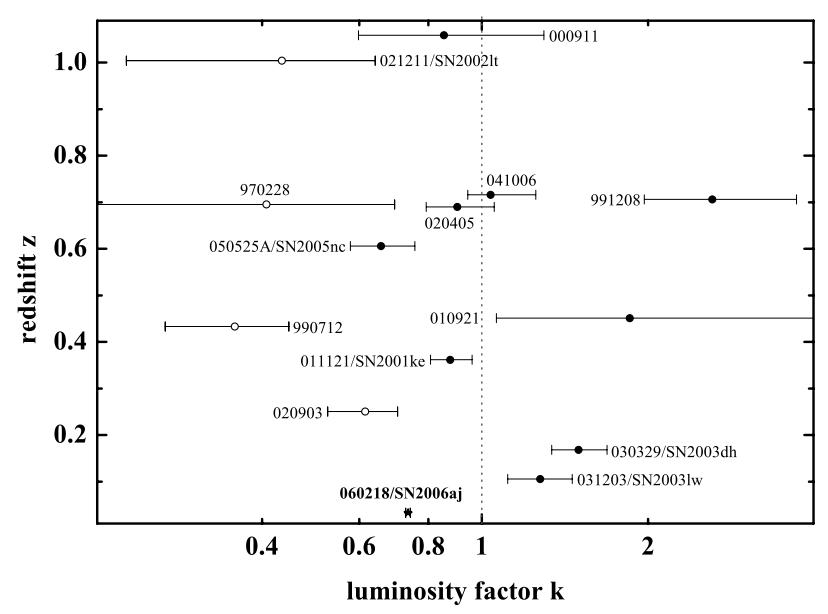

Fig. 4. The distribution of the luminosity factor $k$ in the $R$ band with redshift $z$. There is no apparent correlation with redshift, even though we caution that all the $k$ values refer to the $R$ band in the observer frame, corresponding to different wavelengths in the host frame. Filled symbols mark the $k$ values that have been corrected for host extinction, open symbols represent supernovae for which this correction could not be applied. A star marks the value derived from the $R$-band light curve of SN 2006aj by removing data before 8.8 days from the fit. SN 1998bw is at $k=1$, by definition.

\section{Discussion}

\subsection{SN 2006aj in the context of known GRB-SNe}

SN 2006aj is a rare nearby GRB-SN, with a distance of only about $140 \mathrm{Mpc}$. Its luminosity is about $70 \%$ of that of SN 1998bw, confirming that GRB-selected supernovae may not qualify for the label "standard candle". Given the UVES data of SN 2006aj, taken close to SN maximum light (Guenther et al. 2006; Wiersema et al. 2006), it is unlikely that the deficiency in luminosity is the result of dust extinction in the host galaxy of SN 2006aj.

By the end of 2005, there were 14 optical afterglows ${ }^{7}$ with known redshifts that showed evidence for extra light in the $R$ band at late times (GRBs 970228, 990712, 991208, 000911, 010921, 011121, 020405, 021211, 030329, 031203, 0409248, 041006, 050525A, XRF 020903). For eight afterglows with a known SN bump (GRBs 991208, 000911, 010921, 011121, 020405, 021211, 030329, 041006) K06 were able to derive a host extinction value. We used these to determine the extinctioncorrected luminosities of these $\mathrm{SNe}$ in the observer frame (Fig. 4). It turns out that only two $(030329,031203)$ or perhaps three (including 991208) of the $14 \mathrm{SNe}$ were actually more luminous than SN 1998bw with high significance (but see, e.g., Deng et al. 2005, for a spectroscopic modeling of SN 2003dh that results in $k<1$ ). Remarkably, most extinction corrected SNe cluster around $0.6<k<1.5$. We find that the four SNe not corrected for extinction (GRBs 970228, 990712, 020903, 021211) are typically fainter, implying that a correction for host galaxy extinction will probably also shift them into this range. While one might worry about the fact that for each individual GRB-SN, due to the different redshifts, the $k$ factor refers to a different wavelength region, SN 2006aj does not contradict our assumption that $k$ is not strongly dependent on wavelength (Fig. 5).

\footnotetext{
7 Compared to Z04/Z05, GRB 980703 has been removed as we now have reason to doubt the identification of a SN bump.

8 This event is not included in our study as sufficient photometry has not been published yet.
} 


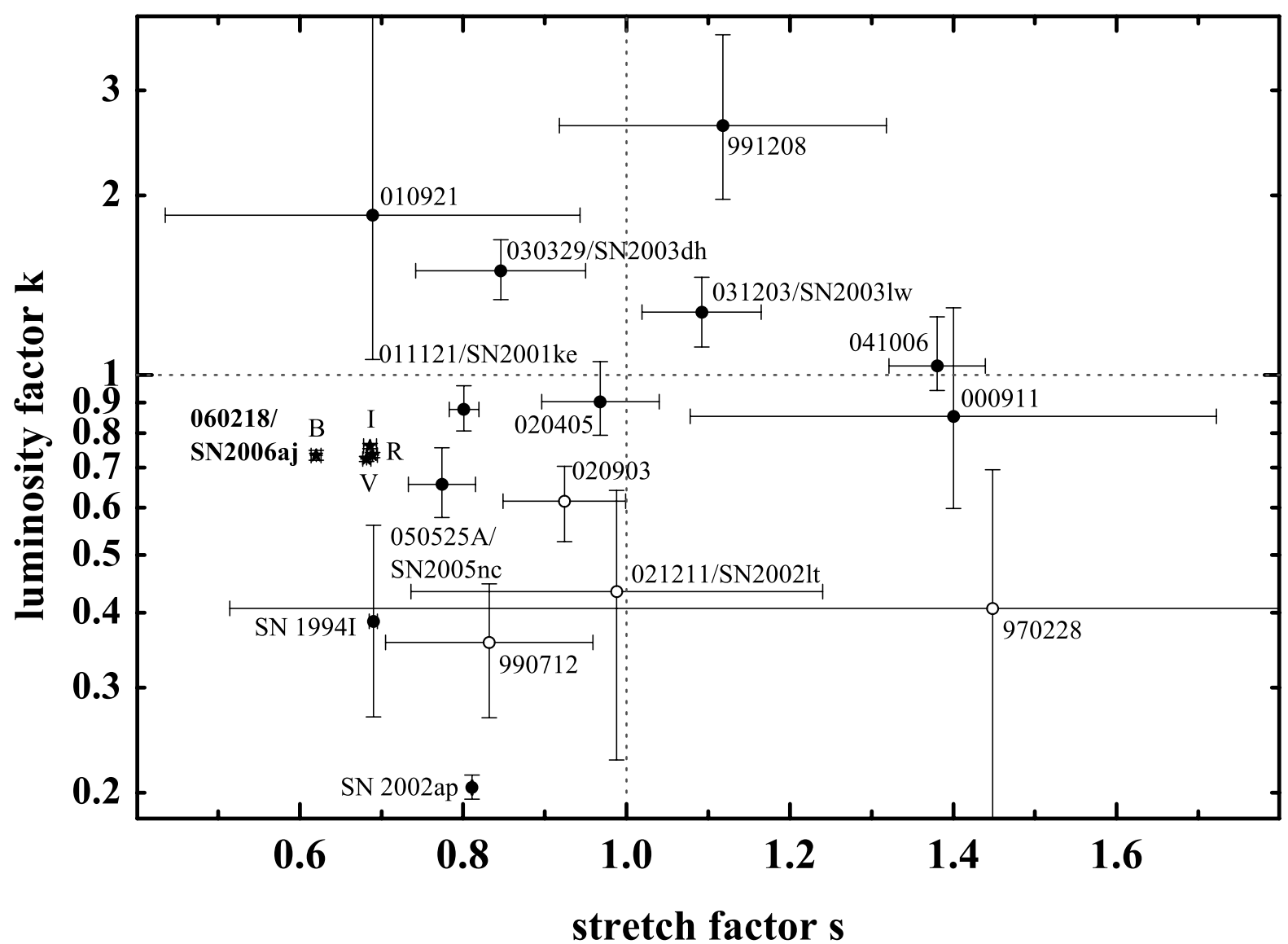

Fig. 5. Luminosity factor $k$ versus stretch factor $s$ in the $R$ band for all GRB-SNe in our sample (Table 3). The symbols are identical to those used in Fig. 4. SN 1998bw defines $k=1, s=1$. Note that in the case of SN 2006aj the values for $B V R I$ are plotted. Furthermore, we plot the type Ic supernova SN 1994I and the type Ic broad-lined SN 2002ap, neither of which are associated with GRBs. Both are fainter than any GRB-SN in our sample for which the host extinction has been corrected.

We conclude that the present data, even though some have large uncertainties, indicate that the width of the GRB-SN luminosity function is at least 2 mag, comparable to what is known for the other types of SNe (cf. Richardson et al. 2002; Richardson et al. 2006, henceforth RBB06). In particular, there is no evidence that the luminosity function evolves with redshift: The width of the luminosity function for $z<0.2$ is comparable to the width at $z \approx 0.7$. It is also much narrower than the distribution of intrinsic afterglow luminosities (K06).

\subsection{SN 2006aj in the context of local stripped-envelope supernovae}

It is also interesting to compare the light curve properties of SN 2006aj and other GRB-SNe with well-studied local strippedenvelope (i.e. types Ib, Ic, IIb) supernovae, as is shown in Fig. 6. Distance moduli and absolute magnitudes for these SNe were taken from RBB06 (who use $H_{0}=60 \mathrm{~km} \mathrm{~s}^{-1} \mathrm{Mpc}^{-1}$ ) and have been transformed to the world model used here $\left(H_{0}=\right.$ $71 \mathrm{~km} \mathrm{~s}^{-1} \mathrm{Mpc}^{-1}$ ) by adding $-5 \log 71 / 60=-0.365 \mathrm{mag}$ to the former and $+0.365 \mathrm{mag}$ to the latter values. Similarly, for the world model used here SN 1998bw is 0.19 mag less luminous than given in Galama et al. (1998), who use $H_{0}=$ $65 \mathrm{~km} \mathrm{~s}^{-1} \mathrm{Mpc}^{-1}$, i.e. $k=1$ corresponds to $M_{\mathrm{V}}=-19.16$. The absolute visual magnitudes of the GRB-SNe in our sample were then calculated according to $M_{\mathrm{V}}^{\mathrm{GRB}-\mathrm{SN}}-M_{\mathrm{V}}^{98 \mathrm{bw}}=-2.5 \log k$, assuming that $k$ is independent of wavelength. Figure 6 shows the result obtained in this way. Most notable is that the ensemble of GRB-SNe is at the bright end of the luminosities of local stripped-envelope supernovae. In other words, the present data indicate that type Ic supernovae with associated (detected) GRBs are on average more luminous in the optical bands than those without detected GRBs. In particular, SN 2006aj is no exception from this rule. We caution, however, that our assumption of wavelength-independence of $k$ could be an oversimplification. The larger the redshift, the more uncertain is the absolute $V$-band magnitude of a GRB-SN derived in this way. This uncertainty is not included in the error bars plotted in Fig. 6.

Finally, we note that an observational bias might affect the interpretation of Fig. 6: the larger the redshift, the more difficult it is to observe less luminous stripped-envelope supernovae.

RBB06 give host extinction values for all 27 events in their sample. Therefore, we investigated whether host extinction of GRB-SNe is different from those of local stripped-envelope supernovae. No substantial differences are apparent (Fig. 7). While it is interesting that SN 2006aj is less affected by host extinction compared to the local sample of type Ic SNe, the current sample is too small to draw reliable conclusions from this finding.

\subsection{The stretch factor}

Finally, we consider the statistics of the stretch factor $s$, which is in some sense more reliable since it is not affected by the extinction issue. First of all, among all known GRB-SNe, SN 2006aj 


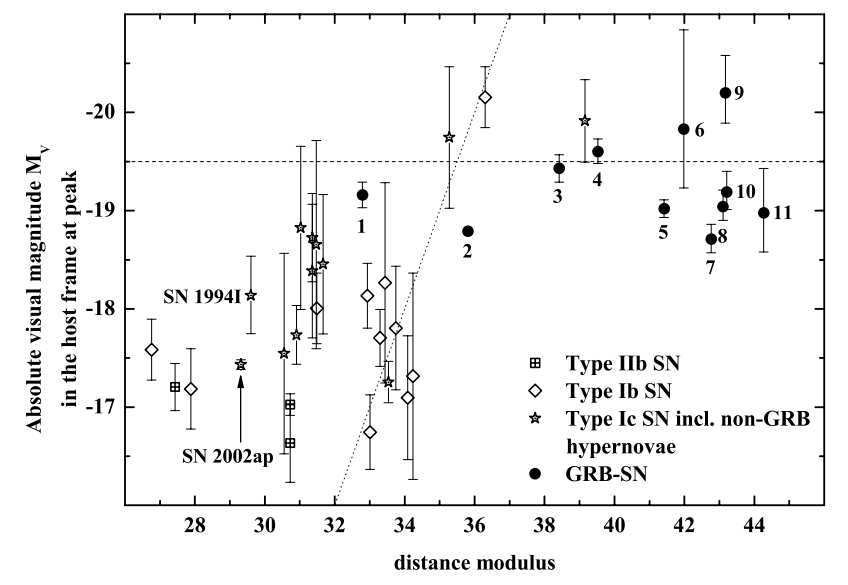

Fig. 6. The absolute $V$-band magnitude $M_{\mathrm{V}}$ of stripped-envelope supernovae versus their distance modulus. This is an expanded version of Figure 1 in RBB06, and the values for SN of type IIb, Ib and Ic have been taken from that work but transformed according to the world model used here. The individual GRB-SNe have the same numbers as in Fig. 7. We have also labeled two local type Ic SNe we have included in our study (Fig. 5; Appendix). GRB-SNe without known host extinction are not included. The dashed line at $M_{\mathrm{V}}=-19.5$ denotes the typical absolute magnitude of type Ia SNe (the "ridgeline"). The slanted dotted line denotes a constant visual magnitude $m_{\mathrm{V}}=16$ in the case of no extinction.

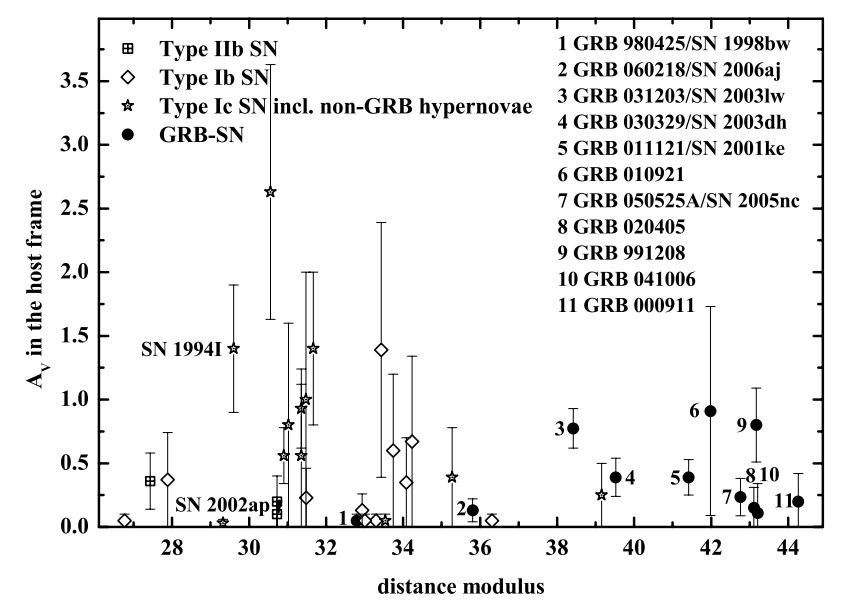

Fig. 7. The visual host-galaxy extinction $A_{\mathrm{V}}$ (in the host frame) of stripped-envelope supernovae versus their distance modulus. The values for SNe of type IIb, Ib and Ic have been taken from RBB06, while the data for the GRB-SNe are from K06. We labeled individual GRB$\mathrm{SNe}$ and the two type Ic SNe we have also studied. GRB-SNe without known host extinction were not included.

has the smallest $s$ value, i.e. it is the fastest GRB-SN ever seen (Pian et al. 2006; Mazzali et al. 2006b; Modjaz et al. 2006a). Interestingly, about half of the GRB-SNe have $s<1$, i.e., they are evolving faster than SN 1998bw. In contrast, the evolution of the SN associated with GRB 041006 was very slow (Fig. 5) with high significance (Stanek et al. 2005). Basically only two SNe (000911, 970228) occupy the $(k<1, s>1)$ region, i.e., they are slow and subluminous, contrary to the general trend, but both have large error bars. Stanek et al. (2005) noted that for GRB-SNe a relation may exist between light curve shape and luminosity, similar to the one established for type Ia SNe (see Fig. 3 in Stanek et al. 2005). Figure 5 neither supports nor contradicts the existence of such a potential relation. While a trend of rising $k$ with rising $s$ seems to be visible, a fit to the data does not support this trend with reasonable statistical significance.

\subsection{Shortcomings of the procedure}

The procedure applied here has shortcomings that are basically related to cosmological effects on one hand and to data quality on the other.

As long as one is only concerned with broad-band photometry, which is the case in this study, the observed light curves of GRB-SNe usually refer to different wavelength bands in their host frames. However for SN 1998bw it has been shown that the light curve shape, the time of the peak flux, and the peak flux itself are a function of wavelength (as is the case for other $\mathrm{SN}$ types, too). Unfortunately, the available photometric data base is in most cases restricted to $R$-band observations (in the observer frame). In order to be able to compare light curves of GRB-SNe that occurred at various redshifts with light curves of SN 1998bw, the simplest and in some sense only useful approach is then to assume that the luminosity ratio $k$ and the stretch factor $s$, which are both normalized to SN 1998bw, are independent of wavelength. In other words, we assume that the SED of all GRB-SNe (in their host frame) is the same at all times.

In addition, the data base used for our light curve fits is usually weighted to data obtained past the peak time of a GRB-SN under consideration. The reason is that before the peak time usually the afterglow dominates the light of the optical transient, while after the peak time usually the SN light dominates. This problem is difficult to overcome. Therefore, for basically all cosmologically remote $\mathrm{SNe}$ we have no information about the details of the SN light curves at early times up to several days after the corresponding burst. In particular the stretch factor $s$ is then mainly affected by the late-time behaviour of the SN light curves.

It is therefore by no means clear if a relation between $s$ and $k$ indeed exists. It is possible that such a relation is hidden by the relatively large error bars for individual $(k, s)$ values of the GRB-SNe on the one hand (Fig. 5) and by the basic assumptions that went into the procedure we applied here on the other hand. More spectral data from GRB-SNe might finally solve this issue. However, progress made in this regard during the last years was only modest, at best. Most Swift detected GRBs are at such a high redshift that no SN spectroscopy can be performed with current telescopes within a reasonable amount of observing time. Therefore, the photometric approach utilized here derives some of its justification from these spectroscopic limitations.

\section{Conclusions}

SN 2006aj is the fastest evolving and one of the least luminous GRB-SN discovered so far, being only about $70 \%$ as luminous as SN 1998bw. This places it at the faint end of the GRB-SN luminosity distribution which so far, after extinction correction, covers the range from about 0.6 to 2 times the luminosity of SN 1998bw. Placing SN 2006aj in the context of the luminosities of local Type Ic supernovae without detected GRB (RBB06) reveals, however, that SN 2006aj is still at the bright end of their luminosity distribution. SN 2006aj thus follows the general "rule" that GRB-SNe tend to be more luminous than (local) Type Ic SNe without detected GRB.

GRBs 980425 and 060218 suggest that the prompt emission properties are not correlated with the optical properties of the associated SNe. GRB 980425 had a duration of $31 \mathrm{~s}$ 
(Pian et al. 1999), while GRB 060218 lasted for more than 2000 s (Campana et al. 2006), and the corresponding isotropic equivalent energy release in the gamma-ray band was different by a factor of 10 . The corresponding SN luminosities, however, differ by only $30 \%$. Assuming that differences in instrumental sensitivities (BeppoSAX versus Swift) do not play a role here, this supports the notion that the properties of the GRB and the associated SN are to a large extent independent of each other.

Acknowledgements. We thank the staff at ESO/Paranal, in particular A. Bik, C. Dumas, O. Hainaut, E. Jehin, C. Ledoux, O. Marco, C. Melo, D. Naef, L. Schmidtobreick for performing the observations. F.P., D.A.K. and S.K. acknowledge support by DFG grant K1 766/13-2 and by the German Academic Exchange Service (DAAD) under grant No. D/05/54048. A.V.F. is supported by NASA/Swift grants NNG05GF35G and NNG06GI86G. KAIT and its ongoing research were made possible by generous donations from Sun Microsystems, Inc., the Hewlett-Packard Company, AutoScope Corporation, Lick Observatory, the National Science Foundation, the University of California, the Sylvia \& Jim Katzman Foundation, and the TABASGO Foundation. This work has benefitted from collaboration within the EU FP6 Research Training Network HPRN-CT2002-00294 "Gamma-Ray Bursts: an Enigma and a Tool." We thank the anonymous referee for a prompt and constructive report.

\section{Appendix A: SN 1994I and SN 2002ap}

Following the suggestion of the referee, we consider here two local type Ic supernovae that were not physically related to a detected GRB but are often used for comparison with GRB-SNe, and place them in the $k, s$ context used in the present paper. While the fit appears to be worse, it should be noted that when fitting a GRB-SN in all cases the fit is practically always weighted to data points lying after the light curve maximum when the SN light dominates the afterglow light.

SN 1994 I in the Whirlpool Galaxy M 51 (Puckett et al. 1994) counts as the "prototypical" type Ic supernova (Filippenko et al. 1995), although it is faster than any other (RBB06). For the analysis of the light curve we used $R$-band data from Yokoo et al. (1994), Schmidt \& Kirschner (1994), Lee et al. (1995) and Richmond et al. (1996) (with late data taken only from Schmidt \& Kirschner and Richmond et al.). SN 1994I occurred near the bulge of M 51 and was highly reddened: Richmond et al. estimate $E_{(B-V)}=0.45 \pm 0.16$ with a Milky Way extinction curve. The explosion date of the supernova is not well known. From light curve modeling, Iwamoto et al. (1994) derive an explosion date of JD 2449433. These authors equate this with a $B$-band maximum 12 days after the explosion. A comparison with the data from Richmond et al. (1996) shows, however, that JD 2449437 corresponds to 12 days before the $B$ maximum. Using the latter, the early light curve becomes strongly subluminous in comparison to SN 1998bw, has to be excluded from the fit and $s=0.69 \pm 0.01$ is found from the location of the maximum alone. On the other hand, if we take March 31.0 UT (JD 2 449 442.5) as the explosion date, we find that up to an age of 25 days the early light curve is well fitted by the SN 1998bw light curve. Assuming a distance modulus of 29.60 mag (Richmond et al. 1996), corresponding to a distance $d=8.34 \mathrm{Mpc}$ for the world model used here, we find, after correcting for the host-galaxy extinction (Richmond et al. 1996), $k=0.39_{-0.12}^{+0.17}$. Thus SN 1994I is fainter than any GRB-SN when we account for host extinction.

SN 2002ap, which exploded in the outer spiral arms of M 74 (Nakano et al. 2002), is the closest and best followed-up broadlined SN Ic to date. A search through data of all IPN satellites did not reveal any GRB that could be associated with the SN (Hurley et al. 2002); still, the explosion date was well constrained, as

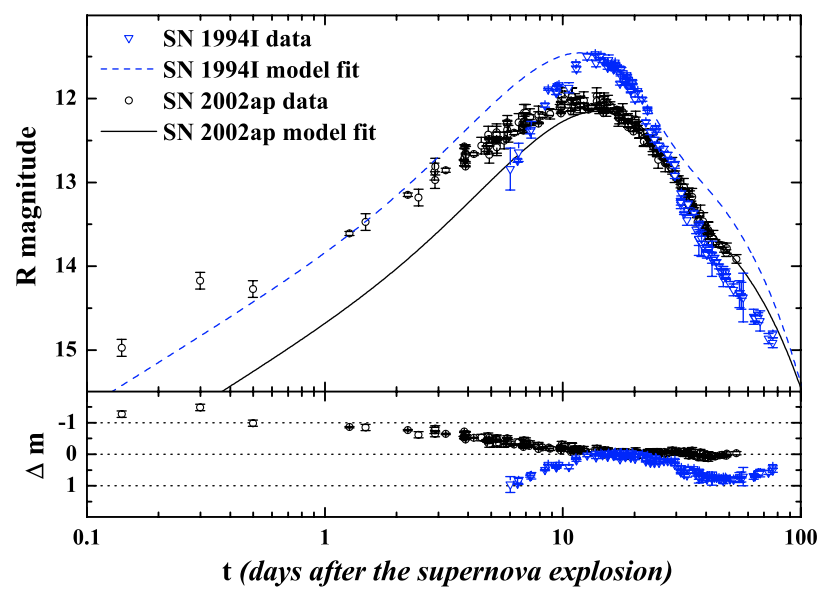

Fig. A.1. The two local type Ic supernovae SN 1994I and SN 2002ap fitted with the model light curve of SN 1998bw at the respective redshifts. In both cases, the light curve of SN 1998bw does not fit the light curves of the other SNe completely. The best agreement is obtained for SN 2002ap, where data after ten days up to 60 days are fitted well by the SN 1998bw light curve. For SN 1994I data before and after the maximum had to be excluded from the fit.

the supernova was discovered very rapidly (Mazzali et al. 2002). For the analysis of the light curve we collected data from the following sources: Gal-Yam et al. (2002), Borisov et al. (2002), Cook et al. (2002), Nishihara et al. (2002), Yoshii et al. (2003), Pandey et al. (2003), Doroshenko et al. (2003), Foley et al. (2003), and Vinkó et al. (2004). In addition we made use of the data collected at SNWEB $^{9}$. Based on these data SN 2002ap is overluminous at early times in comparison to SN 1998bw. The host-galaxy extinction is very small, $E_{(B-V)}=0.020 \pm 0.0008$ (Takada-Hidai et al. 2002). Correcting for this and the Galactic extinction, and assuming a distance modulus of $29.32 \mathrm{mag}$ (Foley et al. 2003), which corresponds to a distance $d=7.3 \mathrm{Mpc}$ for the cosmological model used here, we find $k=0.219 \pm 0.0002$ and $s=0.747 \pm 0.0006$. Thus, the stretch factor $s$ for SN 2002ap is very close to what we find for SN 2006aj, but the SN is much fainter.

\section{References}

Adelman-McCarthy, J. K., Agüeros, M. A., Allam, S. S., et al. 2006, ApJS, 162, 38

Bagoly, Z., Mészáros, A., Balázs, L. G., et al. 2006, A\&A, 453, 797

Bersier, D., Fruchter, A. S., Strolger, L.-G., et al. 2006, ApJ, 643, 284

Borisov, G., Dimitrov, D., Semkov, M., et al. 2002, IBVS, 5264

Bloom, J. S., Kulkarni, S. R., Djorgovski, S. G., et al. 1999, Nature, 401, 453

Blustin, A. J., Band, D., Barthelmy, S., et al. 2006, ApJ, 637, 901

Campana, S., Mangano, V., Blustin, A. J., et al. 2006, Nature, in press [arXiv: astro-ph/0603279]

Cobb, B. E., Bailyn, C. D., van Dokkum, P. G., \& Natarajan, P. 2006, ApJ, 645, L113

Cook, L. M., Katkova, E. V., Sokolov, N. A., \& Guseva, I. S. 2002, IBVS, 5283

Cool, R. J., Eisenstein, D. G., Hogg, D. W., et al. 2006, GCN, 4777

Cusumano, G., Barthelmy, S., Gehrels, N., et al. 2006, GCN, 4775

Deng, J., Tominaga, N., Mazzali, P. A., Maeda, K., \& Nomoto, K. 2005, ApJ, 624,898

Della Valle, M., Malesani, D., Bloom, J. S., et al. 2006, ApJ, 642, L103

Doroshenko, V. T., Sergeev, S. G., Merkulova, N. I., \& Sergeeva, E. A. 2003, Astrophysics, 46(3), 291

Filippenko, A. V. 1997, ARA\&A, 35, 309

Filippenko, A. V., Barth, A. J., Matheson, T., et al. 1995, ApJ, 450, L11

Filippenko, A. V., Li, W. D., Treffers, R. R., \& Modjaz, M. 2001, in SmallTelescope Astronomy on Global Scales, ed. W. P. Chen, C. Lemme, \& B. Paczyński (San Francisco: ASP), 121

\footnotetext{
9 http://www . astrosurf. com/snweb2/2002/02ap/

Q2apMeas.htm
} 
Foley, R. J., Papenkova, M. S., Swift, B. J., et al. 2003, PASP, 115, 1220 Fynbo, J. P. U., Sollerman, J., Hjorth, J., et al. 2004, ApJ, 609, 962

Galama, T. J., Vreeswijk, P. M., van Paradijs, J., et al. 1998, Nature, 395, 670

Gal-Yam, A., Ofek, E. O., \& Shemmer, O. 2002, MNRAS, 332, L73

Ghirlanda, G., Ghisellini, G., \& Lazzati, D. 2004, ApJ, 616, 331

Guenther, E. W., Klose, S., Vreeswijk, P., Pian, E., \& Greiner, J. 2006, GCN, 4863

Hicken, M., Modjaz, M., Challis, P., et al. 2006, GCN, 4898

Hjorth, J., Sollerman, J., Møller, P., et al. 2003, Nature, 423, 847

Hurley, K., Mazets, E., Golenetskii, S., et al. 2002, GCN, 1252

Iwamoto, K., Nomoto, K., Höflich, P., et al. 1994, ApJ, 437, L115

Jakobsson, P., Levan, A., Fynbo, J. P. U., et al. 2006, A\&A, 447, 897

Kann, D. A., Klose, S., \& Zeh, A. 2006, ApJ, 641, 993 (K06)

Landolt, A. U. 1992, AJ, 104, 340

Lee, M. G., Kim, E., Kim, S. C., et al. 1995, JKAS, 28, 31

Li, W., Filippenko, A. V., Chornock, R., \& Jha, S. 2003a, PASP, 115, 844

Li, W., Filippenko, A. V., Chornock, R., \& Jha, S. 2003b, ApJ, 586, L9

Malesani, D., Tagliaferri, G., Chincarini, G., et al. 2004, ApJ, 609, L5

Masetti, N., Palazzi, E., Pian, E., \& Patat, F. 2006, GCN, 4803

Matheson, T., Garnavich, P. M., Stanek, K. Z., et al. 2003, ApJ, 599, 394

Mazzali, P. A., Deng, J., \& Maeda, K. 2004, ApJ, 572, L61

Mazzali, P. A., Deng, J., Pian, E., et al. 2006a, ApJ, 645, 1323

Mazzali, P. A., Deng, J., Nomoto, K., et al. 2006b, Nature, in press [arXiv: astro-ph/0603567]

Modjaz, M., Stanek, K. Z., Garnavich, P. M., et al. 2006a, ApJ, 645, L21

Mirabal, N., \& Halpern, J. P. 2006, GCN, 4792

Mirabal, N., Halpern, J. P., An, D., Thorstensen, J. R., \& Terndrup, D. M. 2006, ApJ, 643, L99

Nakano, S., Kushida, R., Kushida, Y., \& Li, W. 2002, IAU Circ., 7810

Nishihara, E., Kinugasa, K., Hashimoto, O., et al. 2002, Proc. IAU 8th AsianPacific Regional Meeting, Volume II, ed. S. Ikeuchi, J. Hearnshaw, \& T. Hanawa (The Astronomical Society of Japan), 347
Pandey, S. B., Anupama, G. C., Sagar, R., et al. 2003, MNRAS, 340, 375 Patat, F., Cappellaro, E., Danziger, J., et al. 2001, ApJ, 555, 900 Pian, E., Amati, L., Antonelli, L. A., et al. 1999, A\&AS, 138, 463 Pian, E., Mazzali, P., Masetti, N., et al. 2006, Nature, 442, 1011 Puckett, T., Armstron, J., Johnson, W., et al. 1994, IAU Circ., 5961 Richardson, D., Branch, D., Casebeer, D., et al. 2002, AJ, 123, 745 Richardson, D., Branch, D., \& Baron, E. 2006, AJ, 131, 2233 (RBB06) Richmond, M. W., Van Dyk, S. D., Ho, W., et al. 1996, AJ, 111, 327 Schmidt, B. A., \& Kirschner, R. 1994, private communication Soderberg, A. M., Kulkarni, S. R., Price, P. A., et al. 2006, ApJ, 636, 391 Soderberg, A. M., Berger, E., \& Schmidt, B. 2006, IAUC, 8674

Soderberg, A. M., Kulkarni, S. R., Nakar, E., et al. 2006, Nature, in press [arXiv: astro-ph/0604389]

Sollerman, J., Jaunsen, A. O., Fynbo, J. P. U., et al. 2006, A\&A, 454, 503

Spergel, D. N., Verde, L., Peiris, H. V., et al. 2003, ApJS, 148, 175

Stanek, K. Z., Matheson, T., Garnavich, P. M., et al. 2003, ApJ, 591, L17

Stanek, K. Z., Garnavich, P. M., Nutzman, P. A., et al. 2005, ApJ, 626, L5

Stetson, P. B. 1987, PASP, 99, 191

Steele, I. A., Smith, R. J., Rees, P. C., et al. 2004, SPIE, 5489, 679

Takada-Hidai, M., Aoki, W., \& Zhao, G. 2002, PASJ, 54, 899

Vinkó, J., Blake, R. M., Sárneczky, K., et al. 2004, A\&A, 427, 453

Wiersema, K., et al. 2006, in preparation

Woosley, S. E., \& Bloom, J. S. 2006, ARA\&A, in press

Yokoo, T., Arimoto, J., Matsumoto, K., Takahashi, A., \& Sadakane, A. 1994, PASJ, 46, L191

Yoshii, Y., Tomita, H., Kobayashi, Y., et al. 2003, ApJ, 592, 467

Zeh, A., Klose, S., \& Hartmann, D. H. 2004, ApJ, 609, 952 (Z04)

Zeh, A., Klose, S., \& Hartmann, D. H. 2005, in Proc. 22nd Texas Symp. on Relativistic Astrophysics, ed. P. Chen et al. (Stanford: SLAC) [arXiv: astro-ph/0503311] (Z05) 
P. Ferrero et al.: SN 2006aj and GRB supernovae, Online Material p 1

\section{Online Material}


Table 1. The photometry of SN 2006aj from the Very Large Telescope (VLT), the Liverpool Telescope (LT) and the Katzman Automatic Imaging Telescope (KAIT), including the acquisition images for the VLT spectroscopy (Pian et al. 2006). The date is the UT exposure mid-time. Column $t-t_{0}$ refers to the time in days after the burst trigger at $t_{0}=3: 34: 30 \mathrm{UT}$ of Feb. 18, 2006 (Cusumano et al. 2006). The third column gives measured magnitudes which are corrected neither for extinction nor flux from the host galaxy. The fourth column gives the pure supernova magnitudes after correcting for Galactic extinction, host-galaxy flux and host-galaxy extinction. Exposure times are in seconds.

\begin{tabular}{|c|c|c|c|c|c|c|}
\hline Date & $\overline{t-t_{0}}$ & Mag. (measured) & Mag. (corrected) & Exposure & Filter & Telescope \\
\hline February 20.8924 & 2.7434 & $18.63 \pm 0.06$ & $18.14 \pm 0.06$ & $8 \times 150$ & $\bar{B}$ & $\mathrm{LT}$ \\
\hline February 21.0307 & 2.8817 & $18.57 \pm 0.03$ & $18.07 \pm 0.03$ & $2 \times 30$ & $B$ & VLT \\
\hline February 22.1219 & 3.9729 & $18.41 \pm 0.08$ & $17.87 \pm 0.08$ & 450 & $B$ & KAIT \\
\hline February 23.0550 & 4.9060 & $18.24 \pm 0.04$ & $17.68 \pm 0.04$ & $2 \times 30$ & $B$ & VLT \\
\hline February 23.1209 & 4.9719 & $18.29 \pm 0.08$ & $17.74 \pm 0.08$ & 450 & $B$ & KAIT \\
\hline February 24.1218 & 5.9728 & $18.13 \pm 0.08$ & $17.56 \pm 0.08$ & 450 & $B$ & KAIT \\
\hline February 25.0467 & 6.8977 & $18.11 \pm 0.04$ & $17.54 \pm 0.04$ & $2 \times 30$ & $B$ & VLT \\
\hline February 26.0398 & 7.8908 & $18.05 \pm 0.03$ & $17.47 \pm 0.03$ & $2 \times 30$ & $B$ & VLT \\
\hline February 27.0483 & 8.8993 & $18.01 \pm 0.03$ & $17.43 \pm 0.03$ & 60 & $B$ & VLT \\
\hline February 28.0037 & 9.8547 & $18.07 \pm 0.04$ & $17.49 \pm 0.04$ & $2 \times 30$ & $B$ & VLT \\
\hline March 1.0337 & 10.8847 & $18.14 \pm 0.03$ & $17.57 \pm 0.03$ & $2 \times 30$ & $B$ & VLT \\
\hline March 2.0297 & 11.8807 & $18.22 \pm 0.03$ & $17.66 \pm 0.03$ & $2 \times 30$ & $B$ & VLT \\
\hline March 3.0330 & 12.8840 & $18.35 \pm 0.03$ & $17.81 \pm 0.03$ & $2 \times 30$ & $B$ & VLT \\
\hline March 4.0303 & 13.8813 & $18.47 \pm 0.03$ & $17.95 \pm 0.03$ & $2 \times 30$ & $B$ & VLT \\
\hline March 5.0119 & 14.8629 & $18.61 \pm 0.03$ & $18.11 \pm 0.03$ & $2 \times 30$ & $B$ & VLT \\
\hline March 6.0364 & 15.8874 & $18.66 \pm 0.03$ & $18.17 \pm 0.03$ & $2 \times 30$ & $B$ & VLT \\
\hline March 7.8899 & 17.7409 & $18.97 \pm 0.07$ & $18.56 \pm 0.07$ & $5 \times 100$ & $B$ & LT \\
\hline March 8.0332 & 17.8842 & $19.03 \pm 0.03$ & $18.64 \pm 0.04$ & 60 & $B$ & VLT \\
\hline March 8.9983 & 18.8493 & $19.10 \pm 0.03$ & $18.73 \pm 0.04$ & 60 & $B$ & VLT \\
\hline March 10.0009 & 19.8519 & $19.26 \pm 0.04$ & $18.95 \pm 0.05$ & 60 & $B$ & VLT \\
\hline March 11.9977 & 21.8487 & $19.40 \pm 0.04$ & $19.16 \pm 0.05$ & $60+30$ & $B$ & VLT \\
\hline March 13.0145 & 22.8655 & $19.57 \pm 0.05$ & $19.43 \pm 0.07$ & 60 & $B$ & VLT \\
\hline March 13.9976 & 23.8486 & $19.57 \pm 0.05$ & $19.43 \pm 0.07$ & 60 & $B$ & VLT \\
\hline February 20.8787 & 2.7279 & $18.19 \pm 0.06$ & $17.86 \pm 0.06$ & $5 \times 150$ & $\bar{V}$ & LT \\
\hline February 21.0323 & 2.8833 & $18.17 \pm 0.03$ & $17.83 \pm 0.03$ & $2 \times 30$ & $V$ & VLT \\
\hline February 22.1277 & 3.9787 & $17.92 \pm 0.08$ & $17.54 \pm 0.08$ & 300 & $V$ & KAIT \\
\hline February 23.0573 & 4.9083 & $17.80 \pm 0.03$ & $17.41 \pm 0.03$ & $2 \times 30$ & $V$ & VLT \\
\hline February 23.1266 & 4.9776 & $17.77 \pm 0.08$ & $17.37 \pm 0.08$ & 300 & $V$ & KAIT \\
\hline February 24.1275 & 5.9785 & $17.69 \pm 0.08$ & $17.28 \pm 0.08$ & 300 & $V$ & KAIT \\
\hline February 24.8713 & 6.7223 & $17.61 \pm 0.06$ & $17.19 \pm 0.06$ & $5 \times 100$ & $V$ & LT \\
\hline February 25.0483 & 6.8993 & $17.58 \pm 0.03$ & $17.16 \pm 0.03$ & $2 \times 30$ & $V$ & VLT \\
\hline February 26.0413 & 7.8923 & $17.51 \pm 0.03$ & $17.08 \pm 0.03$ & $2 \times 30$ & $V$ & VLT \\
\hline February 26.8720 & 8.7230 & $17.48 \pm 0.06$ & $17.05 \pm 0.06$ & $5 \times 100$ & $V$ & LT \\
\hline February 27.0156 & 8.8666 & $17.45 \pm 0.03$ & $17.02 \pm 0.03$ & 60 & $V$ & VLT \\
\hline February 27.0497 & 8.9007 & $17.46 \pm 0.03$ & $17.03 \pm 0.03$ & 60 & $V$ & VLT \\
\hline February 28.0053 & 9.8563 & $17.45 \pm 0.03$ & $17.02 \pm 0.03$ & $2 \times 30$ & $V$ & VLT \\
\hline March 1.0353 & 10.8863 & $17.45 \pm 0.03$ & $17.02 \pm 0.03$ & $2 \times 30$ & $V$ & VLT \\
\hline March 2.0313 & 11.8823 & $17.47 \pm 0.03$ & $17.04 \pm 0.03$ & $2 \times 30$ & $V$ & VLT \\
\hline March 2.8673 & 12.7183 & $17.50 \pm 0.06$ & $17.07 \pm 0.06$ & $3 \times 100$ & $V$ & LT \\
\hline March 3.0345 & 12.8855 & $17.51 \pm 0.03$ & $17.08 \pm 0.03$ & $2 \times 30$ & $V$ & VLT \\
\hline March 4.0331 & 13.8841 & $17.56 \pm 0.03$ & $17.14 \pm 0.03$ & $2 \times 30$ & $V$ & VLT \\
\hline March 5.0134 & 14.8644 & $17.60 \pm 0.03$ & $17.18 \pm 0.03$ & $2 \times 30$ & $V$ & VLT \\
\hline March 6.0379 & 15.8889 & $17.68 \pm 0.03$ & $17.27 \pm 0.03$ & $2 \times 30$ & $V$ & VLT \\
\hline March 6.8912 & 16.7422 & $17.67 \pm 0.08$ & $17.26 \pm 0.08$ & $2 \times 100$ & $V$ & LT \\
\hline March 8.0018 & 17.8528 & $17.85 \pm 0.03$ & $17.46 \pm 0.03$ & 60 & $V$ & VLT \\
\hline March 8.0347 & 17.8857 & $17.86 \pm 0.03$ & $17.47 \pm 0.03$ & 60 & $V$ & VLT \\
\hline March 8.9998 & 18.8508 & $17.92 \pm 0.03$ & $17.54 \pm 0.03$ & 60 & V & VLT \\
\hline March 9.0067 & 18.8577 & $17.92 \pm 0.03$ & $17.54 \pm 0.03$ & 60 & $V$ & VLT \\
\hline March 10.0024 & 19.8534 & $18.01 \pm 0.03$ & $17.65 \pm 0.03$ & 60 & $V$ & VLT \\
\hline March 10.0080 & 19.8590 & $17.98 \pm 0.03$ & $17.61 \pm 0.03$ & 60 & $V$ & VLT \\
\hline March 11.9987 & 21.8497 & $18.14 \pm 0.03$ & $17.80 \pm 0.03$ & $60+30$ & $V$ & VLT \\
\hline March 13.0157 & 22.8667 & $18.22 \pm 0.04$ & $17.89 \pm 0.04$ & 60 & $V$ & VLT \\
\hline March 13.9991 & 23.8501 & $18.30 \pm 0.03$ & $17.99 \pm 0.03$ & 60 & $V$ & VLT \\
\hline March 14.9992 & 24.8502 & $18.41 \pm 0.03$ & $18.12 \pm 0.03$ & $2 \times 30$ & $V$ & VLT \\
\hline March 16.0023 & 25.8533 & $18.42 \pm 0.05$ & $18.14 \pm 0.05$ & $2 \times 30$ & $V$ & VLT \\
\hline
\end{tabular}


Table 1. continued.

\begin{tabular}{|c|c|c|c|c|c|c|}
\hline$\overline{\text { Date }}$ & $\overline{t-t_{0}}$ & Mag. (measured) & Mag. (corrected) & Exposure & Filter & $\overline{\text { Telescope }}$ \\
\hline February 20.8578 & 2.7088 & $18.10 \pm 0.06$ & $17.88 \pm 0.06$ & $6 \times 150$ & $R$ & $\mathrm{LT}$ \\
\hline February 20.9362 & 2.7872 & $18.10 \pm 0.06$ & $17.88 \pm 0.06$ & $6 \times 150$ & $R$ & LT \\
\hline February 21.0339 & 2.8849 & $18.02 \pm 0.03$ & $17.78 \pm 0.03$ & $2 \times 30$ & $R$ & VLT \\
\hline February 21.0374 & 2.8884 & $18.02 \pm 0.03$ & $17.78 \pm 0.03$ & 30 & $R$ & VLT \\
\hline February 21.1294 & 2.9804 & $17.98 \pm 0.08$ & $17.73 \pm 0.08$ & 120 & $R$ & KAIT \\
\hline February 21.1312 & 2.9822 & $17.96 \pm 0.08$ & $17.71 \pm 0.08$ & 120 & $R$ & KAIT \\
\hline February 21.1330 & 2.9840 & $17.97 \pm 0.08$ & $17.73 \pm 0.08$ & 120 & $R$ & KAIT \\
\hline February 21.1349 & 2.9859 & $17.98 \pm 0.08$ & $17.73 \pm 0.08$ & 120 & $R$ & KAIT \\
\hline February 21.1367 & 2.9877 & $17.96 \pm 0.09$ & $17.71 \pm 0.09$ & 120 & $R$ & KAIT \\
\hline February 22.1317 & 3.9827 & $17.76 \pm 0.08$ & $17.48 \pm 0.08$ & 300 & $R$ & KAIT \\
\hline February 22.1398 & 3.9908 & $17.79 \pm 0.09$ & $17.51 \pm 0.09$ & 120 & $R$ & KAIT \\
\hline February 23.0224 & 4.8734 & $17.66 \pm 0.03$ & $17.37 \pm 0.03$ & 60 & $R$ & VLT \\
\hline February 23.0590 & 4.9100 & $17.66 \pm 0.03$ & $17.37 \pm 0.03$ & $2 \times 30$ & $R$ & VLT \\
\hline February 23.1306 & 4.9816 & $17.60 \pm 0.08$ & $17.30 \pm 0.08$ & 300 & $R$ & KAIT \\
\hline February 23.1387 & 4.9897 & $17.60 \pm 0.10$ & $17.29 \pm 0.10$ & 120 & $R$ & KAIT \\
\hline February 24.1315 & 5.9825 & $17.50 \pm 0.09$ & $17.19 \pm 0.09$ & 300 & $R$ & KAIT \\
\hline February 24.1395 & 5.9905 & $17.50 \pm 0.10$ & $17.19 \pm 0.10$ & 120 & $R$ & KAIT \\
\hline February 24.8645 & 6.7155 & $17.44 \pm 0.07$ & $17.12 \pm 0.07$ & $5 \times 100$ & $R$ & LT \\
\hline February 25.0499 & 6.9009 & $17.40 \pm 0.04$ & $17.08 \pm 0.04$ & $2 \times 30$ & $R$ & VLT \\
\hline February 26.0429 & 7.8939 & $17.31 \pm 0.04$ & $16.98 \pm 0.04$ & $2 \times 30$ & $R$ & VLT \\
\hline February 26.8677 & 8.7187 & $17.29 \pm 0.06$ & $16.96 \pm 0.06$ & $3 \times 100$ & $R$ & LT \\
\hline February 27.0512 & 8.9022 & $17.28 \pm 0.03$ & $16.95 \pm 0.03$ & 60 & $R$ & VLT \\
\hline February 28.0068 & 9.8578 & $17.25 \pm 0.03$ & $16.91 \pm 0.03$ & $2 \times 30$ & $R$ & VLT \\
\hline March 1.0370 & 10.8880 & $17.23 \pm 0.03$ & $16.89 \pm 0.03$ & $2 \times 30$ & $R$ & VLT \\
\hline March 2.0328 & 11.8838 & $17.23 \pm 0.03$ & $16.89 \pm 0.03$ & $2 \times 30$ & $R$ & VLT \\
\hline March 2.8604 & 12.7114 & $17.26 \pm 0.06$ & $16.93 \pm 0.06$ & $5 \times 100$ & $R$ & LT \\
\hline March 3.0360 & 12.8870 & $17.24 \pm 0.03$ & $16.90 \pm 0.03$ & $2 \times 30$ & $R$ & VLT \\
\hline March 3.8601 & 13.7111 & $17.25 \pm 0.07$ & $16.91 \pm 0.07$ & $2 \times 100$ & $R$ & LT \\
\hline March 4.0346 & 13.8856 & $17.26 \pm 0.03$ & $16.93 \pm 0.03$ & $2 \times 30$ & $R$ & VLT \\
\hline March 5.0148 & 14.8658 & $17.24 \pm 0.04$ & $16.90 \pm 0.04$ & $2 \times 30$ & $R$ & VLT \\
\hline March 6.0082 & 15.8592 & $17.33 \pm 0.03$ & $17.00 \pm 0.03$ & 60 & $R$ & VLT \\
\hline March 6.0394 & 15.8904 & $17.33 \pm 0.03$ & $17.00 \pm 0.03$ & $2 \times 30$ & $R$ & VLT \\
\hline March 6.8834 & 16.7344 & $17.36 \pm 0.07$ & $17.03 \pm 0.07$ & $4 \times 100$ & $R$ & LT \\
\hline March 7.8841 & 17.7341 & $17.41 \pm 0.06$ & $17.09 \pm 0.06$ & $5 \times 100$ & $R$ & LT \\
\hline March 8.0361 & 17.8871 & $17.46 \pm 0.03$ & $17.14 \pm 0.03$ & 60 & $R$ & VLT \\
\hline March 9.0012 & 18.8522 & $17.56 \pm 0.03$ & $17.26 \pm 0.03$ & 60 & $R$ & VLT \\
\hline March 10.0038 & 19.8548 & $17.60 \pm 0.03$ & $17.30 \pm 0.03$ & 60 & $R$ & VLT \\
\hline March 11.9997 & 21.8507 & $17.62 \pm 0.04$ & $17.32 \pm 0.04$ & $60+30$ & $R$ & VLT \\
\hline March 13.0169 & 22.8679 & $17.68 \pm 0.03$ & $17.39 \pm 0.03$ & 60 & $R$ & VLT \\
\hline March 14.0013 & 23.8523 & $17.89 \pm 0.03$ & $17.63 \pm 0.03$ & 60 & $R$ & VLT \\
\hline February 21.0358 & 2.8868 & $17.86 \pm 0.03$ & $17.80 \pm 0.03$ & $2 \times 30$ & $I$ & VLT \\
\hline February 22.1357 & 3.9867 & $17.69 \pm 0.08$ & $17.60 \pm 0.08$ & 300 & $I$ & KAIT \\
\hline February 23.0604 & 4.9114 & $17.54 \pm 0.03$ & $17.41 \pm 0.03$ & $2 \times 30$ & $I$ & VLT \\
\hline February 23.1346 & 4.9856 & $17.49 \pm 0.09$ & $17.36 \pm 0.09$ & 300 & $I$ & KAIT \\
\hline February 24.1356 & 5.9866 & $17.31 \pm 0.09$ & $17.15 \pm 0.09$ & 300 & $I$ & KAIT \\
\hline February 25.0514 & 6.9024 & $17.25 \pm 0.03$ & $17.08 \pm 0.03$ & $2 \times 30$ & $I$ & VLT \\
\hline February 26.0444 & 7.8954 & $17.16 \pm 0.03$ & $16.98 \pm 0.03$ & $2 \times 30$ & $I$ & VLT \\
\hline February 27.0526 & 8.9036 & $17.10 \pm 0.03$ & $16.91 \pm 0.03$ & 60 & $I$ & VLT \\
\hline February 28.0083 & 9.8593 & $17.05 \pm 0.04$ & $16.85 \pm 0.04$ & $2 \times 30$ & $I$ & VLT \\
\hline March 1.0386 & 10.8896 & $17.04 \pm 0.03$ & $16.84 \pm 0.03$ & $2 \times 30$ & $I$ & VLT \\
\hline March 2.0328 & 11.8854 & $17.03 \pm 0.03$ & $16.83 \pm 0.03$ & $2 \times 30$ & $I$ & VLT \\
\hline March 3.0376 & 12.8886 & $17.03 \pm 0.03$ & $16.83 \pm 0.03$ & $2 \times 30$ & $I$ & VLT \\
\hline March 4.0362 & 13.8872 & $17.07 \pm 0.04$ & $16.87 \pm 0.04$ & $2 \times 30$ & $I$ & VLT \\
\hline March 5.0165 & 14.8675 & $17.01 \pm 0.06$ & $16.81 \pm 0.06$ & $2 \times 30$ & $I$ & VLT \\
\hline March 6.0410 & 15.8920 & $17.08 \pm 0.03$ & $16.89 \pm 0.03$ & $2 \times 30$ & $I$ & VLT \\
\hline March 9.0026 & 18.8536 & $17.21 \pm 0.03$ & $17.03 \pm 0.03$ & 60 & $I$ & VLT \\
\hline March 10.0052 & 19.8562 & $17.26 \pm 0.03$ & $17.09 \pm 0.03$ & 60 & $I$ & VLT \\
\hline March 11.9993 & 21.8503 & $17.35 \pm 0.05$ & $17.19 \pm 0.05$ & $60+30$ & $I$ & VLT \\
\hline March 13.0181 & 22.8691 & $17.37 \pm 0.05$ & $17.21 \pm 0.05$ & 60 & $I$ & VLT \\
\hline March 14.0027 & 23.8537 & $17.48 \pm 0.03$ & $17.34 \pm 0.03$ & 60 & $I$ & VLT \\
\hline
\end{tabular}

